\title{
Efficiency and Security Trade-Off in Supporting Range Queries on Encrypted Databases ${ }^{\star}$
}

\author{
Jun Li and Edward R. Omiecinski \\ College of Computing, \\ Georgia Insitute of Technology, \\ 801 Atlantic Drive, Atlanta, GA 30332 \\ \{junli, edwardo\}@cc.gatech.edu
}

\begin{abstract}
The database-as-a-service (DAS) model is a newly emerging computing paradigm, where the DBMS functions are outsourced. It is desirable to store data on database servers in encrypted form to reduce security and privacy risks since the server may not be fully trusted. But this usually implies that one has to sacrifice functionality and efficiency for security. Several approaches have been proposed in recent literature for efficiently supporting queries on encrypted databases. These approaches differ from each other in how the index of attribute values is created. Random one-to-one mapping and order-preserving are two examples. In this paper we will adapt a prefix-preserving encryption scheme to create the index. Certainly, all these approaches look for a convenient trade-off between efficiency and security. In this paper we will discuss the security issues and efficiency of these approaches for supporting range queries on encrypted numeric data.
\end{abstract}

\section{Introduction}

The database-as-a-service (DAS) model [1] is a new computing paradigm that has emerged recently. To save cost, data storage and management are outsourced to database service providers. In other words, highly sensitive data are now stored in locations that are not under the data owner's control, such as leased space and partners' sites. This can put data confidentiality at risk. Therefore, it is desirable to store data in encrypted form to protect sensitive information. Also queries may reveal private information about the user [2]. In this paper, we discuss how to efficiently support searching functionality, in particular, range queries, while preserving data confidentiality and user privacy. The motivation within this model of processing is to provide security and privacy but also have the database service provider do most of the query processing. Several approaches have been proposed to generate the index that enables queries to be processed against encrypted data with different levels of efficiency and security [3/5[6]. In this paper, we will adapt a prefix-preserving encryption scheme to create the

\footnotetext{
^ This work has been supported in part by National Science Fundation Grant CCR0121643.
}

S. Jajodia and D. Wijesekera (Eds.): Data and Applications Security 2005, LNCS 3654, pp. 69-83 2005.

(C) IFIP International Federation for Information Processing 2005 
index. We will also discuss the security issues and efficiency of these approaches for supporting range queries on encrypted data.

One simple way to preserve the confidentiality is to decrypt the data when performing search. There are several drawbacks with this approach. First, all the data stored in the database needs to be decrypted for every query. Second, this approach assumes the server is secure and fully trusted. This assumption is less justified in the DAS paradigm.

A major type of database queries is range-based, composed of intervals in the underlying domain of the attributes. Attributes such as name, not typically thought of as numerical, can be indexed and therefore linearized in some fashion. In this paper we will mainly be concerned with interval-matching or exact-matching as query conditions. Interval-matching is defined as a boolean function $f_{[a, b]}(x)$, which returns true if and only if $x \in[a, b]$. Because computers can handle only inherently finite and discrete attribute values, one can assume without loss of generality $x, a$ and $b$ are all nonnegative integers. Exact-matching is a special case of interval-matching in which $a$ is equal to $b$.

The paper is organized as the follows, in Sect. 2, we survey the related work and discuss possible solutions based on well-known mechanisms. Section 3 shows how a relation is encrypted and stored on the server. In Sect. 4, we present a scheme that efficiently supports interval-matching as query conditions. First we show how an interval-matching problem can be transformed into a set of prefix-matching problems. Then the prefix-preserving encryption algorithm is presented. At the end of the section, we describe that with prefix-preserving encryption how a condition in a range query is translated to a condition over server-side representation and how select operations are implemented. Section 5 analyzes one possible attack against the random one-to-one mapping scheme and the prefix-preserving scheme, while in Sect.6] we have some additional discussion on the security of the prefix-preserving scheme. Section 7 compares the prefixpreserving scheme with the random one-to-one mapping scheme in the aspects of client side cost, server side cost and communication cost for supporting range queries. We then conclude the paper in Sect. 8.

\section{Related Work}

Recently providing security and privacy in DAS has drawn considerable attention 3 4 516]. The bucket index technique proposed in [36] relies on partitioning attribute domains of a client's table into sets of buckets. The index value of each remote table attribute value is the bucket number to which the corresponding plain value belongs. This representation supports efficient evaluation of both exact-matching and interval-matching predicates on the database service provider; however, it makes it awkward to manage the correspondence between bucket numbers and the actual attribute values present in the database. For the convenience of comparison, in the rest of this paper, when we discuss about this approach, we will assume that the size of each bucket is 1 and the bucket number is generated by a random one-to-one mapping of the plaintext value. In 
this case, the server will not return any redundant data to the client. Therefore, the client does not need any database functionality to filter out unsolicited data. This fulfills the goal of the DAS model, i.e., outsourcing database management and having the database server do most of the work.

In [4, the authors quantitatively evaluate the level of inference exposure associated with the publication of attribute indexes generated by a random one-toone mapping. In the solution they propose for supporting interval-based queries, the task of determining $\mathrm{B}^{+}$-tree information is left to the customer. The advantage of their solution is that the content of $\mathrm{B}^{+}$-tree is not visible to an untrusted database service provider. The disadvantage is that a lot of data processing has to occur on client machines. This mitigates the advantage of the DAS model.

In [7, a sequence of strictly increasing polynomial functions is used for encrypting integer values while preserving their order. In [5], another form of order-preserving encryption is provided for computing the index. It takes a userprovided target distribution as input and transform the plaintext values in such a way that the transformation preserves the order while the transformed values follow the target distribution. The authors assume an application environment where the goal is safety from an adversary who has access to all (but only) encrypted values (the so called ciphertext only attack [8]). In this paper, we will not only examine the prefix-preserving scheme under ciphertext only attack, but also examine it under known plaintext attack [8] (i.e., an adversary is assumed to gain full knowledge to certain number of 〈plaintext, ciphertext〉 pairs through means other than compromising the key).

All of the aforementioned schemes including the scheme proposed in this paper suffer from a same problem, i.e., they preserve statistics. That is, an adversary may know exactly how many entries each value has, even though the plaintexts of the indexes themselves are unknown. This can lead the adversary into an easier inference. Elovici et al. proposed an index scheme that does not reveal database statistics 9. However, it assumes that the cell coordinates (including Table ID, Row ID, and Column ID) are stable. That is, insert, update and delete operations do not change the coordinates of existing cells. This puts additional restrictions to the implementation of the DBMS.

A potential technique that can support searching on encrypted data is computing with encrypted data [10. However, an expensive protocol between clients and database service providers is needed. A closely related topic is Private Information Retrieval (PIR) [2]. PIR mechanisms allow clients to query databases without revealing which entries are of interest. PIR schemes often require multiple non-colluding servers, consume large amounts of bandwidth, and do not guarantee the confidentiality of the data.

\section{Data Organization}

In a relational DBMS, data are organized in tables (e.g., the Employee data in Table 1, where the underlined attribute represents the key of the table). The database can be encrypted with regard to different units, which can be individual 
Table 1. Employee

\begin{tabular}{|c|c|c|c|c|c|}
\hline FNAME & LNAME & SSN & ADDRESS & SALARY & DNO \\
\hline \hline John & Smith & 123456789 & 731 Fondren, Storrs, CT & 30000 & 5 \\
\hline Franklin & Wong & 333445555 & 638 Voss, Storrs, CT & 40000 & 5 \\
\hline Alicia & Zelaya & 999887777 & 3321 Castle, Storrs, CT & 25000 & 4 \\
\hline Ahmad & Jabbar & 987987987 & 980 Dallas, Storrs, CT & 25000 & 5 \\
\hline James & Borg & 888665555 & 450 Stone, Storrs, CT & 55000 & 1 \\
\hline
\end{tabular}

Table 2. Encrypted_Employee

\begin{tabular}{|c|c|c|c|}
\hline Enc_tuple & $I_{\text {SSN }}$ & $I_{\text {SALARY }}$ & $I_{D N O}$ \\
\hline \hline fjftejcCcWsGqfChXcHuRzoriODCRxvD & 068764019 & 6488 & 250 \\
\hline tprJMmfjXJNs74fZZfL1TridemjZnWvY & 277737042 & 45639 & 250 \\
\hline edVI8JvVSjmzXsrmDIiosZabdFnnorwy & 080581877 & 53798 & 224 \\
\hline z4tzGJUdsyy7Eb0puESatLCXOXckVTWA & 203690710 & 53798 & 250 \\
\hline zzdqGlqngQgwJurSqsyFrejiia6KCNMk & 929644962 & 20577 & 59 \\
\hline
\end{tabular}

table, a column of a table, a row (tuple) of a table or a given column within each row (i.e., the data item value). Encrypting at a coarser level of granularity such as a table implies that the entire table must be returned as the result of a query, although encryption/decryption will be more efficient. Encrypting at a finer level such as a data item allows for more efficient query processing but requires increased overhead for encryption/decryption [1. As in 3456], we assume encryption to be performed at the tuple level. To provide the server with the ability to select a set of tuples to be returned in response to a query, we associate each encrypted tuple with a number of indexing attributes. An index can be associated with each attribute in the original relation on which conditions need to be evaluated for query processing.

Each plaintext relation will be stored as a relation with one attribute representing the encrypted tuple and additional attributes representing the indexes. Each plaintext tuple $t\left(A_{1}, \ldots, A_{n}\right)$ is mapped onto a tuple $t^{\prime}\left(E(t), I_{1}, \ldots, I_{m}\right)$ where $m \leq n$. The attribute $E(t)$ stores an encrypted string that corresponds to the entire plaintext tuple, and each $I_{i}$ corresponds to the index over some $A_{j}$. The encryption function $E$ is treated as a black box in our discussion. Any block cipher such as AES [11, DES [12] etc., can be used to encrypt the tuples. Table 2 illustrates an example of the corresponding encrypted/indexed relation Encrypted_Employee where Enc_tuple contains the encrypted tuples, while $I_{S S N}, I_{S A L A R Y}$, and $I_{D N O}$ are indexes over attributes SSN, SALARY, and DNO respectively.

\section{A Prefix-Preserving Encryption Based Scheme}

\subsection{Transforming Interval-Matching into Prefix-Matching}

In this section, we will transform interval-matching into prefix-matching. Prefixmatching has been used widely in databases and networks. The transformation 
is based on the fact that an arbitrary interval can be converted into a union of prefix ranges, where a prefix range is one that can be expressed by a prefix [13]. For example, the interval $[32,111]$, the 8 -bit binary representation of which is [00100000, 01101111], can be represented by a set of prefixes $\{001 *, 010 *, 0110 *\}$. Throughout this paper, the notation $*$ is used to denote an arbitrary suffix. To verify that a number is in the interval is equivalent to check that the number matches any of those prefixes in the set. For example, 37 (00100101 in binary) is in the interval as it matches prefix 001*, while 128 (10000000 in binary) is not in the interval since it matches none of those three prefixes.

Let $n$ denote the length of the binary representation of the data, and let $p_{n}$ denote the number of prefixes needed to represent an interval. We have the following theorem on the upper bound of $p_{n}$.

Theorem 1. For any interval $\left[a_{1} a_{2} \cdots a_{n}, b_{1} b_{2} \cdots b_{n}\right](n \geq 2), p_{n} \leq 2(n-1)$.

The proof of this theorem is omitted here due to the lack of space and can be found in our technical report [14. Note that for interval $\left[1,2^{n}-2\right]$, it can be easily verified that $p_{n}$ is equal to $2(n-1)$. Therefore, the upper bound is tight.

Theorem 2. For a given $n$, considering all possible intervals $\left[a_{1} a_{2} \cdots a_{n}\right.$, $\left.b_{1} b_{2} \cdots b_{n}\right]$, if we assume all the intervals appear with the same probability, i.e., all queries are equi-probable, the average number of $p_{n}$ is equal to $\frac{(n-2) 2^{2 n-1}+(n+1) 2^{n}+1}{2^{2 n-1}+2^{n-1}}$, which is approximately equal to $n-2$, when $n$ is large.

The proof of this theorem is omitted here due to the lack of space and can be found in our technical report 14. From these two theorems we see that the upper bound of $p_{n}$ is a linear function of $n$ and the average number of $p_{n}$ is approximately a linear function of $n$. This is a very nice feature.

In Fig. 11 we present a recursive algorithm to generate the set of prefixes for a given interval $\left[a_{1} a_{2} \cdots a_{n}, b_{1} b_{2} \cdots b_{n}\right]$.

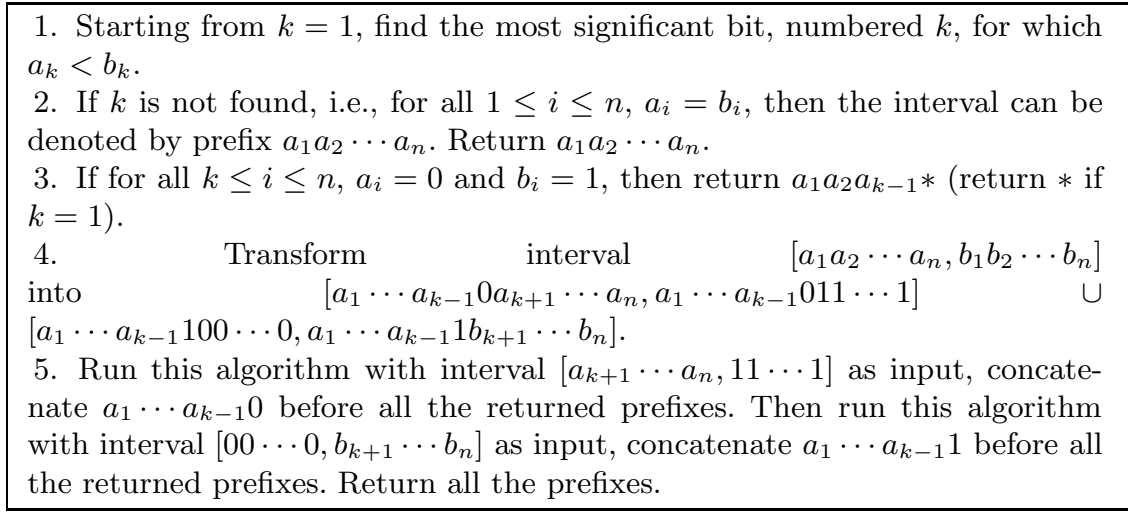

Fig. 1. The algorithm for transforming interval $\left[a_{1} a_{2} \cdots a_{n}, b_{1} b_{2} \cdots b_{n}\right]$ into prefixes 
We have seen that matching an interval based on a set of prefix-matchings is both simple and efficient. Therefore prefix-preserving encryption algorithm can be used to efficiently support interval-matching as a query condition while preserving the confidentiality of data and queries.

\subsection{Prefix-Preserving Encryption}

After transforming interval-matching into prefix-matching, we need a prefixpreserving encryption scheme to generate the index, so that the database system will be able to answer the queries based on encrypted data and queries. We apply an encryption scheme proposed by $\mathrm{Xu}$ et al. [15] for prefix-preserving IP address anonymization.

Definition 1. (Prefix-preserving encryption) ([15]) We say that two $n$-bit numbers $a=a_{1} a_{2} \cdots a_{n}$ and $b=b_{1} b_{2} \cdots b_{n}$ share a $k$-bit prefix $(0 \leq k \leq n)$, if $a_{1} a_{2} \cdots a_{k}=b_{1} b_{2} \cdots b_{k}$, and $a_{k+1} \neq b_{k+1}$ when $k<n$. An encryption function $E_{p}$ is defined as a one-to-one function from $\{0,1\}^{n}$ to $\{0,1\}^{n}$. An encryption function $E_{p}$ is said to be prefix-preserving, if, given two numbers a and $b$ that share a $k$-bit prefix, $E_{p}(a)$ and $E_{p}(b)$ also share a $k$-bit prefix.

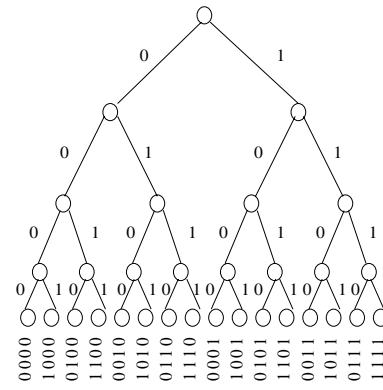

(a) plaintext tree

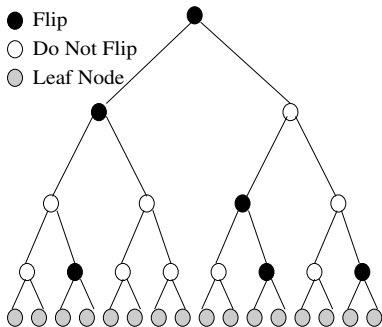

(b) encryption function

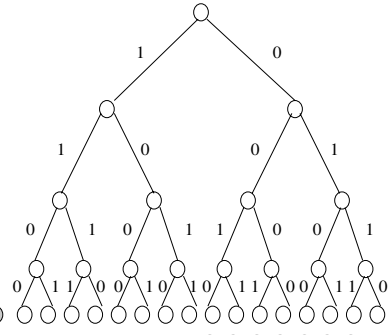

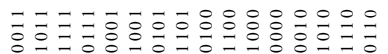

(c) ciphertext tree

Fig. 2. An example of prefix-preserving encryption

It is helpful to consider a geometric interpretation of prefix-preserving encryption [15. If a plaintext can take any value of a $n$-bit number, the entire set of plaintexts can be represented by a complete binary tree of height $n$. This is called the plaintext tree. Each node in the plaintext tree (excluding the root node) corresponds to a bit position, indicated by the height of the node, and a bit value, indicated by the direction of the branch from its parent node. Figure 2(a) shows a plaintext tree (using 4-bit plaintexts for simplicity).

A prefix-preserving encryption function can be viewed as specifying a binary variable for each non-leaf node (including the root node) of the plaintext tree. This variable specifies whether the encryption function "flips" this bit or not. Applying the encryption function results in the rearrangement of the plaintext tree into a ciphertext tree. Figure 2(c) shows the ciphertext tree resulting from 
the encryption function shown in Fig. 2(b). Note that an encryption function will, therefore, consist of $2^{n}-1$ binary variables, where $n$ is the length of a plaintext.

A general form of prefix-preserving encryption function is presented in [15]. Let $f_{i}$ be a function from $\{0,1\}^{i}$ to $\{0,1\}$, for $i=1,2, \cdots, n-1$ and $f_{0}$ is a constant function. Given a plaintext $a=a_{1} a_{2} \cdots a_{n}$, the ciphertext $a_{1}^{\prime} a_{2}^{\prime} \cdots a_{n}^{\prime}$ will be computed by the algorithm given in Fig. 3 . According to Theorem 1 (canonical form theorem) in [15, the algorithm given in Fig. 3] is a prefix-preserving encryption algorithm.

1. Compute $a_{i}^{\prime}$ as $a_{i} \oplus f_{i-1}\left(a_{1} a_{2} \cdots a_{i-1}\right)$, where $\oplus$ stands for the exclusive-or operation, for $i=1,2, \cdots, n$.

2. Return $a_{1}^{\prime} a_{2}^{\prime} \cdots a_{n}^{\prime}$.

Fig. 3. Prefix-preserving encryption algorithm

In [15], the prefix-preserving encryption scheme is defined as instantiating functions $f_{i}$ with cryptographically strong stream ciphers or block ciphers as follows:

$$
f_{i}\left(a_{1} a_{2} \cdots a_{i}\right):=\mathcal{L}\left(\mathcal{R}\left(a_{1} a_{2} \cdots a_{i}, \kappa\right)\right)
$$

where $i=1, \cdots, n-1$ and $\mathcal{L}$ returns the "least significant bit". Here $\mathcal{R}$ is a pseudorandom function or a pseudorandom permutation (i.e., a block cipher). $\kappa$ is the cryptographic key used in the pseudorandom function $\mathcal{R}$. Its length should follow the guideline specified for the pseudorandom function that is actually adopted.

The encryption function can be performed quickly as it only involves $n-1$ symmetric key cryptographic operations, and these $n-1$ operations can be done in parallel. A prefix expresses a prefix range, thus a prefix-matching query can be efficiently processed as a range query with a $\mathrm{B}^{+}$-tree index structure. We will compare the performance of the prefix-preserving scheme with the random one-to-one mapping scheme in Sect. 7.

\subsection{Implementing Range Queries over Encrypted Relations}

With the prefix-preserving encryption algorithm, denoted by $E_{p}$, we can translate specific query conditions in operations (such as selects and joins) to corresponding conditions over server-side representation. This translation function is called $M a p_{\text {cond }}$. Since this paper is mainly focused on supporting range queries, we will only consider select operations in this paper. This scheme can handle other relational operations as well. The discussion is omitted here due to the lack of space and can be found in our technical report [14.

A select query condition is a boolean expression specified on relation attributes. It can be made up of a number of clauses of the form

$<$ attribute $><$ comparison op $><$ value $>$, 
where $<$ attribute $>$ is the name of an attribute, < comparison op $>$ is one of the operations $\{=, \leq, \geq\}$, and $<$ value $>$ is a constant value from the attribute domain. Clauses can be arbitrarily connected by the boolean operators AND, OR, and NOT to form a general query condition. It has been discussed in [3] how to translate a composite condition to the corresponding condition over serverside representation after each clause is translated. Hereafter we discuss how to translate a single clause.

attribute = value: Since the prefix-preserving encryption is a one-to-one mapping, the mapping is simply defined as follows:

$\operatorname{Map}_{\text {cond }}\left(A_{i}=v\right) \Rightarrow E_{p}\left(A_{i}\right)=E_{p}(v)$.

attribute $\leq$ value (attribute $\geq$ value): A query condition $A_{i} \leq v\left(A_{i} \geq\right.$ $v)$ is equivalent to an interval-matching of $f_{\left[v_{\text {min }}, v\right]}\left(A_{i}\right)\left(f_{\left[v, v_{\max }\right]}\left(A_{i}\right)\right)$, where $v_{\min }\left(v_{\max }\right)$ is the lower (upper) bound of the attribute domain. The interval $\left[v_{\min }, v\right]\left(\left[v, v_{\max }\right]\right)$ can be converted into a union of prefix ranges, $\left\{P_{1}, P_{2}, \cdots\right.$, $\left.P_{l}\right\}$, with Algorithm 1. Therefore, interval-matching $f_{\left[v_{m i n}, v\right]}\left(A_{i}\right)$ can be transformed to a set of prefix-matchings $\left\{M_{P_{1}}\left(A_{i}\right), M_{P_{2}}\left(A_{i}\right), \cdots, M_{P_{l}}\left(A_{i}\right)\right\}\left(M_{P_{k}}\left(A_{i}\right)\right.$ denotes the boolean function, which returns true if and only if the value of $A_{i}$ matches prefix $P_{k}$ ). Then the prefix-preserving encryption can be applied on the prefixes. Therefore, the mapping is defined as follows:

$\operatorname{Map}_{\text {cond }}\left(A_{i} \leq v\right) \Rightarrow$

$\left\{M_{E_{p}\left(P_{1}\right)}\left(E_{p}\left(A_{i}\right)\right)\right.$ OR $M_{E_{p}\left(P_{2}\right)}\left(E_{p}\left(A_{i}\right)\right)$ OR $\cdots$ OR $\left.M_{E_{p}\left(P_{l}\right)}\left(E_{p}\left(A_{i}\right)\right)\right\}$.

Consider a select operation $\sigma_{C}(R)$ on a relation $R$, where $C$ is a condition specified on one or more of the attributes $A_{1}, A_{2}, \cdots, A_{n}$ of $R$. The operation can be rewritten as follows:

$\sigma_{C}(R)=D\left(\sigma_{\text {Map }_{\text {cond }}(C)}(E(R))\right.$,

where $E(R)$ is the encrypted relational table (e.g., the Encrypted_Employee table presented in Table 2), and $D$ is the corresponding decryption function of $E$. The operation $\sigma_{\text {Map }_{\text {cond }}(C)}(E(R))$ will be executed at the server. The results will be transmitted to the client. The client then can get the query results by applying decryption function $D$.

\section{Attack with a Set of Queries}

In this section, we will discuss the security issues of the prefix-preserving scheme proposed in this paper and the schemes proposed in the literature, i.e., random one-to-one mapping and order-preserving. There are many possible attacks against these schemes [45. We are not going to examine all the possible attacks, instead we will discuss a particular one, which can be applied to both the random one-to-one mapping scheme and the prefix-preserving scheme.

An adversary may compromise the confidential information by gathering query predicate conditions. Sometimes it is reasonable for an adversary to assume that the index set against one attribute from each query may be derived from a single interval. In other words, each index set, though contains multiple indexes, represents only a single interval. Based on this assumption, the encryption mapping may be revealed partially, i.e., the adversary can figure out a coarse order of a set of indexes. This will be further explained in the rest of this section. 
The feasibility of this attack is constrained by the ability of adversaries to collect enough queries. Furthermore, clients may not obey the assumption, i.e., they may not always submit a single interval-matching for each predicate against one index attribute in a query. This will complicate the attack as well.

To alleviate this problem, clients may specify different keys to generate indexes for different attributes, thus preventing an adversary from aggregating information from different attributes. Also, clients can inject some noise into their queries to undermine the adversary's assumption. But the price paid is that the clients will receive some data that are not of interest. This compromises the purpose of the DAS model, since the clients still need certain database functionality to be able to filter out redundant results.

The order-preserving encryption preserves the order of plaintexts, so it is trivial for the adversary to figure out the order of any set of indexes generated from the order-preserving scheme. In the remainder of this section we will analyze possible attacks against the random one-to-one mapping scheme and the prefixpreserving scheme.

\subsection{Against the Random One-to-one Mapping Scheme}

An adversary is assumed to be able to collect a set of queries. In each query there is a tuple of index sets. Based on our assumption, the indexes in a set should represent a single interval. Assume the size of the index domain is $2^{n}$. If the adversary is able to collect all the $2^{n}-1$ two-index sets which contain two consecutive indexes, then he/she will be able to figure out an order of all the indexes, but without knowing whether it is an ascending or descending order. If the adversary knows at least one plaintext/ciphertext pair, then he/she will be able to decrypt any index. For example, when $n=2$, if the adversary is able to collect 3 index sets, $\{a, b\},\{b, c\},\{c, d\}$, the he/she will be able to figure out an order of the indexes, a, b, c, d, without knowing if it is an ascending or descending order.

An algorithm to collect two-index sets from a list of index sets is given in Fig. 4 .

1. Discard one-index sets.

2. For any two sets $A, B$ in the list of index sets, if none of $A \cap B, A \cap \bar{B}$, $\bar{A} \cap B$ is an empty set, then add these sets into the new list of index sets (Note that any of these resulted sets still represents a single interval).

3. If any new set is added, go to step 1. Otherwise, collect all two-index sets.

Fig. 4. The algorithm for attacking queries against the random one-to-one mapping

\subsection{Against the Prefix-Preserving Scheme}

To better illustrate the attack against the prefix-preserving scheme, we introduce a definition as follows. 
Definition 2. Given two $k$-bit $(k \geq 2)$ encrypted prefixes $a=a_{1} a_{2} \cdots a_{k} *$ and $b=b_{1} b_{2} \cdots b_{k} *$, if there exists an $i, 1 \leq i \leq k-1$ such that $a_{i} \neq b_{i}$ and the range of $a$ and $b$ can be merged into a single interval, then we call the set of prefixes $\{a, b\}$ a non-trivial two-prefix set with length $k$. We call an encrypted two-prefix set $\left\{a_{1} a_{2} \cdots a_{k-1} a_{k} *, a_{1} a_{2} \cdots a_{k-1} \overline{a_{k}} *\right\}$ a trivial two-prefix set.

It is easy to see that if an adversary has all $\left(2^{k-1}-1\right)$ non-trivial twoprefix sets of length $k$, then he/she will be able to create an order for all encrypted $k$-bit prefixes without knowing if it is an ascending or descending order. If the adversary knows at least one plaintext/ciphertext pair, then he/she will be able to decrypt any encrypted $k$-bit prefix. For example, if an adversary has the following three non-trivial two-prefix sets of length $3,\left\{a_{1} a_{2} a_{3} *, a_{1} \overline{a_{2}} b_{3} *\right\}$, $\left\{a_{1} \overline{a_{2}} \overline{b_{3}} *, \overline{a_{1}} b_{2} c_{3} *\right\},\left\{\overline{a_{1}} b_{2} \overline{c_{3}} *, \overline{a_{1}} \overline{b_{2}} d_{3} *\right\}$, then he/she will be able to figure out the following order for all 3-bit prefixes: $a_{1} a_{2} \overline{a_{3}}, a_{1} a_{2} a_{3} *, a_{1} \overline{a_{2}} b_{3} *, a_{1} \overline{a_{2}} \overline{b_{3}} *, \overline{a_{1}} b_{2} c_{3} *$, $\overline{a_{1}} b_{2} \overline{c_{3}} *, \overline{a_{1}} \overline{b_{2}} d_{3} *, \overline{a_{1}} \overline{b_{2} d_{3}} *$ without knowing whether it is an ascending or descending order. If the plaintext of $a_{1} a_{2} a_{3} *$ is known to be $001 *$, the adversary will be able to decrypt any encrypted 3 -bit prefix.

An adversary is assumed to be able to collect a set of queries. In each query there is a tuple of encrypted prefix sets. Based on our assumption, the encrypted prefixes in a set should represent a single interval. An algorithm to collect nontrivial two-prefix sets of length $k$ from a list of encrypted prefix sets is given in Fig. 5].

1. Preprocess the encrypted prefix sets.

- For any prefix longer than $k$-bit, $a_{1} a_{2} \cdots a_{k} a_{k+1} \cdots a_{l} *(l>k)$, replace it by $a_{1} a_{2} \cdots a_{k} *$.

- For any prefix shorter than $k$-bit, $a_{1} a_{2} \cdots a_{l} *(l<k)$, replace it by all the $k$-bit prefixes which share the $l$-bit prefix.

(Note that after the preprocessing, the encrypted prefixes in a set still represent a single interval)

2. Discard trivial two-prefix sets and one-prefix sets.

3. For any two sets $A, B$ in the list of prefix sets, if none of $A \cap B, A \cap \bar{B}$, $\bar{A} \cap B$ is an empty set, then add these sets into the new list of prefix sets (Note that any of these resulted sets still represents a single interval).

4. If any new set is added, go to step 2. Otherwise, collect all non-trivial two-prefix sets.

Fig. 5. The algorithm for attacking queries against the prefix-preserving scheme

Hereafter we give a simple example of this attack. Suppose an adversary wants to attack the 3 -bit prefixes, and he/she has the following encrypted prefix sets from the queries. $A=\left\{a_{1} a_{2} a_{3} *, a_{1} \overline{a_{2}} *, \overline{a_{1}} b_{2} *, \overline{a_{1}} \overline{b_{2}} b_{3} *\right\}, B=\left\{a_{1} \overline{a_{2}} c_{3} *\right.$, $\left.\overline{a_{1}} b_{2} *, \overline{a_{1}} \overline{b_{2}} b_{3} *\right\}, C=\left\{\overline{a_{1}} b_{2} d_{3} a_{4} *, \overline{a_{1}} \overline{b_{2}} *\right\}$. The adversary can get the following $3-$ bit prefix sets. $A^{\prime}=\left\{a_{1} a_{2} a_{3} *, a_{1} \overline{a_{2}} c_{3} *, a_{1} \overline{a_{2} c_{3}} *, \overline{a_{1}} b_{2} d_{3} *, \overline{a_{1}} b_{2} \overline{d_{3}} *, \overline{a_{1}} \overline{b_{2}} b_{3} *\right\}, B^{\prime}=$ 
$\left\{a_{1} \overline{a_{2}} c_{3} *, \overline{a_{1}} b_{2} d_{3} *, \overline{a_{1}} b_{2} \overline{d_{3}} *, \overline{a_{1}} \overline{b_{2}} b_{3} *\right\}, C^{\prime}=\left\{\overline{a_{1}} b_{2} d_{3} *, \overline{a_{1}} \overline{b_{2}} b_{3} *, \overline{a_{1}} \overline{b_{2} b_{3}} *\right\}$. Then he/ she will get the following non-trivial two-prefix sets: $A^{\prime} \cap \overline{B^{\prime}}=\left\{a_{1} a_{2} a_{3} *, a_{1} \overline{a_{2} c_{3}} *\right\}$, $A^{\prime} \cap C^{\prime}=\left\{\overline{a_{1}} b_{2} d_{3} *, \overline{a_{1}} \overline{b_{2}} b_{3} *\right\}, B^{\prime} \cap \overline{C^{\prime}}=\left\{a_{1} \overline{a_{2}} c_{3} *, \overline{a_{1}} b_{2} \overline{d_{3}} *\right\}$. Finally the adversary will be able to figure out the following order of the 3-bit prefixes: $a_{1} a_{2} \overline{a_{3}} *$, $a_{1} a_{2} a_{3} *, a_{1} \overline{a_{2} c_{3}} *, a_{1} \overline{a_{2}} c_{3} *, \overline{a_{1}} b_{2} \overline{d_{3}}, \overline{a_{1}} b_{2} d_{3} *, \overline{a_{1}} \overline{b_{2}} b_{3} *, \overline{a_{1}} \overline{b_{2} b_{3}} *$, without knowing whether it is an ascending or descending order.

\section{Additional Security Analysis for Prefix-Preserving Encryption}

In this section we will have more discussion about the security of the prefixpreserving encryption scheme. It has been proved that with the instantiating functions as (1) the prefix-preserving encryption scheme is indistinguishable from a random prefix-preserving function, a function uniformly chosen from the set of all prefix-preserving functions when the adversaries are assumed to be computationally bounded. This is elaborated in [15. Moreover, as mentioned in Sect.4.2, when plaintexts can take any value of a $n$-bit number, the prefix-preserving encryption function consists of $2^{n}-1$ binary variables. Therefore, we have a key of $2^{2^{n}-1}$ possibilities. For example, when $n$ is only 16 , the number of possible keys is $2^{65535}$. Therefore, the key $\kappa$ in (11) can be sufficiently long such that it is impractical for adversaries to try each possible key to compromise the prefix-preserving scheme.

In the remainder of this section, we discuss another possible way in which the prefix-preserving scheme may be attacked. An adversary is assumed to have compromised (gain full knowledge to) certain number of (plaintext, ciphertext) pairs through means other than compromising the key, i.e., the known plaintext attack model [8]. Then he/she will be able to infer information from other ciphertexts by prefix-matching, because the encryption is prefix-preserving. For example, if an adversary knows 〈plaintext, ciphertext $\rangle$ pair $\left\langle a_{1} a_{2} \cdots a_{n}, a_{1}^{\prime} a_{2}^{\prime} \cdots a_{n}^{\prime}\right\rangle$, then given another ciphertext $a_{1}^{\prime} a_{2}^{\prime} \cdots a_{k-1}^{\prime} \overline{a_{k}^{\prime}} b_{k+1}^{\prime} \cdots b_{n}^{\prime}$, he/she knows the $k$-bit prefix of the plaintext should be $a_{1} a_{2} \cdots a_{k-1} \overline{a_{k}}$. Note that if an adversary knows one 〈plaintext, ciphertext $\rangle$ pair $\left\langle a_{1} a_{2} \cdots a_{n}, a_{1}^{\prime} a_{2}^{\prime} \cdots a_{n}^{\prime}\right\rangle$, then he/she should also know the $\langle$ plaintext, ciphtertext $\rangle$ pair $\left\langle a_{1} a_{2} \cdots \overline{a_{n}}, a_{1}^{\prime} a_{2}^{\prime} \cdots \overline{a_{n}^{\prime}}\right\rangle$. Therefore, an adversary always knows an even number of 〈plaintext, ciphertext〉 pairs.

Suppose an adversary knows 2 pairs of 〈plaintext, ciphertext $\rangle$. Given a random ciphertext, let $A(n)$ denote the average length of the prefix that can be inferred by prefix-matching, where $n$ is the length of the binary representation of the data. The probability that the $k$-bit prefix of the plaintext can be inferred is $\frac{1}{2^{k}}$, for $1 \leq k \leq n-1$, while for $k=n$, the probability is $\frac{2}{2^{n}}$. Therefore, $A(n)=\sum_{i=1}^{n-1} \frac{i}{2^{i}}+\frac{2 n}{2^{n}}=\sum_{i=0}^{n-1} \frac{1}{2^{i}}=2-\frac{1}{2^{n-1}}<2$. In other words, on the average an adversary can infer no more than 2 bits from a random ciphertext, if he/she knowns 2 pairs of 〈plaintext, ciphertext $\rangle$.

${ }^{1}$ We are assuming the plaintext is uniformly distributed. The information leaked by the known-plaintext attack can be significantly higher, if we consider that the possible values are not uniformly distributed. 
We also analyze the situation that an adversary knows $2 k(k>1)$ pairs of 〈plaintext, ciphertext〉 in our technical report [14. In summary, when $n \rightarrow$ $\infty$, given a ciphertext, the average length of the prefix that can be inferred is bounded by $\log _{2} k+2$ based on numerical results. So the prefix information an adversary can obtain by comparing a ciphtertext against a few pairs of 〈plaintext, ciphertext $\rangle$ is limited. Therefore, we claim that the prefix-preserving scheme is secure even if a few pairs of 〈plaintext, ciphertext) are known by an adversary. To make the system even more secure, the data owner may specify different keys to generate indexes for different attributes, thus preventing an adversary from aggregating information from different attributes.

\section{Performance Comparison}

\subsection{Communication Cost}

With the prefix-preserving scheme, the total length of the indexes for an intervalmatching query condition is less than $2 n(n-1)$ bits. With random one-to-one mapping, the total length is $l \cdot n$, where $l$ is the length of the interval. So when $l$ is larger than $2(n-1)$, the prefix-preserving scheme is more efficient. If we assume all the intervals appear with the same probability, the average length of the interval is $\frac{\sum_{i=1}^{2^{n}} \frac{i(i+1)}{2}}{\sum_{i=1}^{2^{n}} i}=\frac{2^{3 n-1}+3 \cdot 2^{2 n-1}+2^{n}}{3\left(2^{2 n-1}+2 n-1\right)}$, which is approximately equal to $2^{n} / 3$, when $n$ is large. Therefore, the average number of bits of the indexes is about $n \cdot 2^{n} / 3$, which is much greater than $2 n(n-1)$, when $n$ is large.

\subsection{Client Side Cost}

During the encryption of the database, it costs more to use the prefix-preserving scheme to compute the indexes for the records. Since normally the length of the index attribute is smaller than the block size of a typical block cipher, to compute one index, the prefix-preserving encryption will require $n-1$ block cipher encryptions. In contrast, the random one-to-one mapping will require only 1 encryption. Similarly, to encrypt an exact matching query condition, it costs more with prefix-preserving encryption. However, to encrypt an intervalmatching query condition, with prefix-preserving, at most $2(n-1)^{2}$ encryptions are needed. With random one-to-one mapping, the number of encryptions needed is equal to the length of the interval $l$. So when $l$ is larger than $2(n-1)^{2}$, the prefix-preserving scheme is more efficient. If we assume all intervals appear with same probability, then the average length of the interval is about $2^{n} / 3$, which is larger than $2(n-1)^{2}$, when $n$ is larger than 8 .

\subsection{Server Side Cost}

As for the server side cost, we will be mainly concerned about the cost of disk accesses for executing an interval-matching query, since in most cases it is the bottleneck. To estimate the cost of disk accesses, we must know the number of the records $(r)$, and the number of blocks $(b)$ (or close estimates of them). Also, we 


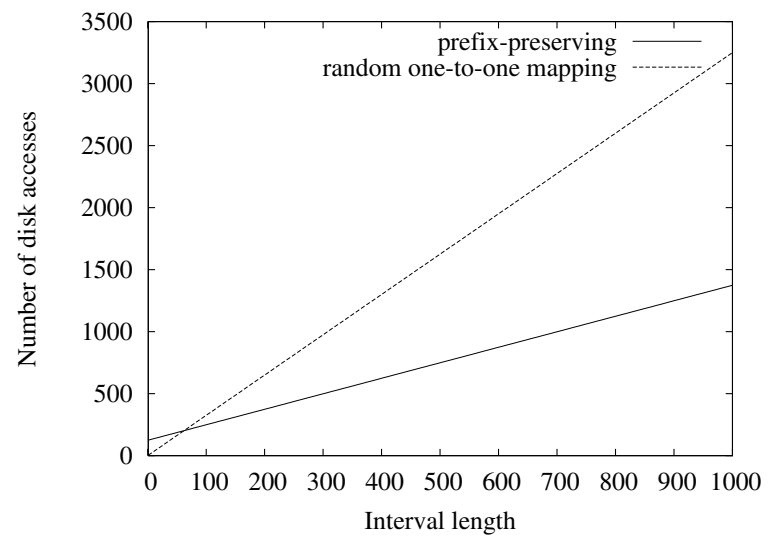

Fig. 6. Number of disk accesses by varying the length of the interval

need to know the number of levels $(h)$ of $\mathrm{B}^{+}$-tree, which is the typical database storage structure for indexes. Another important parameter is the selectivity $(s l)$ of an attribute, which is the fraction of records satisfying an exact-matching condition on the attribute.

Without any index structure, to do a sequential scan of the whole database table, the cost of disk accesses is $b \cdot t_{s}$, where $t_{s}$ is the time needed for a sequential disk block access. In the random one-to-one mapping scheme, with a $\mathrm{B}^{+}$-tree index structure the number of disk accesses needed for retrieving the indexes is $l \cdot h$, where $l$ is the length of the interval, and the number of disk accesses needed for retrieving the actual records is $l \cdot f \cdot s l \cdot b$, where $f$ is the percentage of the values in the interval that actually exist in the table. Therefore, the total timing cost of disk accesses is $l(h+f \cdot s l \cdot b) \cdot t_{r}$, where $t_{r}$ is the time needed for a random disk block access. As mentioned in Sect.4.2 a prefix-matching query can be processed as a range query. In the prefix-preserving scheme, with a $\mathrm{B}^{+}$-tree index structure the number of disk accesses needed for retrieving the indexes is less than $2(n-1)(h-1)+l$, and the number of disk accesses for accessing the actual records is $l \cdot f \cdot s l \cdot b$. So the timing cost of disk accesses is less than $(2(n-1)(h-1)+l+l \cdot f \cdot s l \cdot b) t_{r}$. Therefore, when $l>2(n-1)$, the prefixpreserving scheme is more efficient then the random one-to-one mapping scheme, if sequential scan is not needed. A typical value of $n$ could be 32 . Then when $l>62$, the prefix-preserving scheme is more efficient. A typical disk block size is $32 \mathrm{~K}$ bits. A typical size of a pointer to a disk block is 32 bits. Assume the number of records $r$ in the database is $1 \mathrm{M}$, i.e., $2^{20}$. Then the order of the $\mathrm{B}^{+}$-tree should be $32 \mathrm{~K} /(32+32)=2^{9}$, and the height $h$ should be $\log _{2^{9}} 2^{20}=3$. Figure 6 shows the number of disk accesses with random one-to-one mapping/prefix-preserving, assuming $f$ to be $100 \%, s l=1 / r=2^{-20}$, and the total number of disk blocks in the database $b$ is $256 \mathrm{~K}$. Note that, assuming $t_{r}=64 \cdot t_{s}$, for the random one-to-one mapping scheme, it will be more efficient to do a sequential scan of 
Table 3. Performance comparison of prefix-preserving and random one-to-one mapping for supporting an interval-matching query

\begin{tabular}{|l||l|l|l|}
\hline & $\begin{array}{l}\text { Average communication } \\
\text { cost (length of indexes) }\end{array}$ & $\begin{array}{l}\text { Average client side cost } \\
\text { (number of encryptions) }\end{array}$ & $\begin{array}{l}\text { Server side cost (num- } \\
\text { ber of disk accesses) }\end{array}$ \\
\hline \hline Prefix-preserving & $\leq 2 n(n-1)$ bits & $\leq 2(n-1)^{2}$ & $\begin{array}{l}\leq 2(n-1)(h-1)+l+ \\
l \cdot f \cdot s l \cdot b\end{array}$ \\
\hline Random mapping & $n \cdot 2^{n} / 3$ bits & $2^{n} / 3$ & $l(h+f \cdot s l \cdot b)$ \\
\hline
\end{tabular}

the whole database table when $l>1260$. With the prefix preserving scheme, it might be more efficient to do a sequential scan when $l>3177$.

In summary, we present the communication cost, client side and server side cost for supporting an interval-matching query with random one-to-one mapping /prefix-preserving in Table 3 .

\section{Conclusions}

This paper discusses concerns about protecting sensitive information of data and queries from adversaries in the DAS model. Data and queries need to be encrypted, while the database service provider should be able to efficiently answer queries based on encrypted data and queries. Several approaches are studied in this paper, random one-to-one mapping and prefix-preserving. Possible attacks against these approaches and the performance of these approaches are investigated. The prefix-preserving scheme is more efficient than the random one-to-one mapping scheme for supporting interval-matching queries. In terms of communication cost, with prefix-preserving the length of indexes for an interval is less than $2 n(n-1)$ bits, while with random one-to-one mapping the average is about $n \cdot 2^{n} / 3$ bits. In terms of client side cost, with prefix-preserving the number of encryptions needed is less than $2(n-1)^{2}$, while with random one-to-one mapping the average is about $2^{n} / 3$. In terms of server side cost, the number of disk accesses with the prefix-preserving scheme is smaller than the random one-toone mapping scheme, when the length of the interval is larger than $2(n-1)$. However, the prefix-preserving scheme is less secure than the random one-to-one mapping scheme, because of the constraint of prefix-preserving. For example, with the prefix-preserving encryption a coarse ordering of the encrypted data can be determined by a grouping based on a $k$-bit prefix, but not with a random one-to-one mapping.

Acknowledgement. The authors would like to thank the anonymous referees for their insightful and constructive comments, and Professor Alexandra Boldyreva for her technical comments. 


\section{References}

1. Hacigumus, H., Iyer, B., Mehrotra, S.: Providing database as a service. In: Proceedings of ICDE'02. (2002) 29-38

2. Chor, B., Goldreich, O., Kushilevitz, E., Sudan, M.: Private information retrieval. In: Proceedings of the 36th IEEE Symposium on Foundations of Computer Science. (1995) 41-50

3. Hacigumus, H., Iyer, B., Li, C., Mehrotra, S.: Executing SQL over encrypted data in the database-service-provider model. In: Proceedings of the 2002 ACM SIGMOD International Conference on Management of Data. (2002) 216-227

4. Damiani, E., Vimercati, S.D.C., Jajodia, S., Paraboschi, S., Samarati, P.: Balancing confidentiality and efficiency in untrusted relational dbmss. In: Proceedings of CCS'03. (2003) 93-102

5. Agrawal, R., Kiernan, J., Srikant, R., Xu, Y.: Order preserving encryption for numeric data. In: Proceedings of the 2004 ACM SIGMOD International Conference on Management of Data. (2004) 563-574

6. Hore, B., Mehrotra, S., Tsudik, G.: A privacy-preserving index for range queries. In: Proceedings of VLDB'04. (2004) 720-731

7. Ozsoyoglu, S.C.G., Singer, D., Chung, S.S.: Anti-tamper databases: Querying encrypted databases. In: Proceedings of the 17th Annunal IFIP WG11.3 Working Conference on Database and Application Security. (2003)

8. Stinson, D.R.: Cryptography: Theory and Practice. CRC Press (2002)

9. Elovici, Y., Waisenberg, R., Shmueli, E., Gudes, E.: A structure preserving database encryption scheme. In: Secure Data Management. (2004) 28-40

10. Feigenbaum, J.: Encrypting problem instances, or ..., can you take advantage of someone without having to trust him? In: Proceedings of CRYPTO'85. (1986) 477-488

11. AES: Advanced encryption standard. FIPS 197, Computer Security Resource Center, National Institute of Standards and Technology (2001)

12. DES: Data encryption standard. FIPS PUB 46, Federal Information Processing Standards Publication (1977)

13. Srinivasan, V., Varghese, G., Suri, S., Waldvogel, M.: Fast and scalable layer four switching. In: Proceedings of ACM SIGCOMM'98. (1998) 191-202

14. Li, J., Omiecinski, E.R.: Efficiency and security trade-off in supporting range queries on encrypted databases. Technical Report GIT-CC-05-01, College of Computing, Georgia Institute of Technology (2005) Available at ftp://ftp.cc.gatech.edu/pub/coc/tech_reports/2005/GIT-CC-05-01.pdf.

15. Xu, J., Fan, J., Ammar, M.H., Moon, S.B.: Prefix-preserving IP address anonymization: measurement-based security evaluation and a new cryptographybased scheme. In: Proceedings of the 10th IEEE International Conference on Network Protocols. (2002) 280-289 